\title{
Congrès PSY - Compte-rendu du colloque
}

\author{
Berne, 5 - 7 septembre 2018 \\ Peter Schulthess
}

Le Congrès PSY a été organisé par la Société Suisse de psychiatrie et psychothérapie (SSPP) en partenariat avec l'ASP, la FSP et la SBAP. 1.200 personnes ont participé, une douzaine à peine sont des membres de l'ASP malheureusement. La grande partie des participants venaient de la SSPP dont les congrès annuels attirent toujours près de 1.000 psychiatres. Pourquoi si peu de personnes de notre association? La dominance de la psychiatrie a-t-elle effrayé ? L'exposition industrielle avec de nombreux stands du secteur de la pharma était en réalité plutôt dissuasive pour les psychothérapeutes psychologues. Nous voulons d'autant plus rendre compte du congrès dans à jour! afin que la base des membres soit aussi informée de son contenu. En effet, en plus des présentations concentrées en psychiatrie et pharma, il y a eu aussi de précieuses contributions sur la psychothérapie.

En complément de ce qui a déjà été mentionné dans le compte-rendu de la présidente, j’ai fait part d'autres manifestations.

Dès le début du congrès, Peter Ventevogel de United Nations High Commissioner for Refugees (UNHCR) est intervenu à Genève sur le thème de la "Mental Health of Refugees in Global Perspective». 1 pour cent de la population mondiale serait concernée par une dislocation forcée, il y aurait 22,5 millions de réfugié(e)s. La plupart viendraient d'états aux bas revenus, dans lesquels il y aurait aussi peu de ressources pour les soins de la santé mentale. Labsence d'offres dans les pays pauvres et dans les zones en guerre exigerait beaucoup plus que des connaissances spécialisées et des techniques de professionnels. Trois développements se dessineraient :

1. des approches en lien avec la communauté, qui utilisent des ressources d'auto-assistance et renforcent le lien social.

2. des interventions en psychothérapie brève qui sont aussi utilisées par des assistantes et assistants non professionnels que l'on forme et supervise.

3. intégration des soins de santé mentale dans les soins généraux des réfugiés.

En soirée, à la suite de l'inauguration officielle du congrès, Dirk Helbling, professeur au département des sciences informatiques, a donné une conférence sur «l'utilisation de l'informatique cognitive dans la psychothérapie : Opportunités et risques " lors de la séance de l'ETH. Ces déclarations étaient tellement explosives qu’elles ont été spécialement résumées dans la rubrique «Focus » de ce cahier. Il est passé d'abord par le monde de la collecte des mégadonnées (cf. aussi la contribution de Marianne Roth sur les Fake news) jusqu'à la collecte de données dans le secteur de la santé en général, et pour finir jusqu'aux applications dans la psychothérapie et a abordé les opportunités et risques de cet univers de données qui nous attend.

L'exposé en plénière de Katharina Domschke, Fribourg-en-Brisgau, sur le thème de « l'épigénétique - le deuxième code dans les maladies psychiques » fut également passionnant. L'épigénétique est la spécialité de la biologie qui s'intéresse aux facteurs qui définissent l'activité d'un gène et donc temporairement le développement de la cellule. Les mécanismes épigénétiques comme la méthylation de l'ADN focalisent de plus en plus l'attention en tant que " charnier » biochimique entre «nature » et « nurture » dans la psychiatrie et la psychologie. En effet, l'épigénétique permet dans des changements environnementaux même subtils d'avoir accès à notre patrimoine génétique, une nouvelle étude montre que l'apparition de maladies ou la modification de traits de la personnalité pourraient être influencées d'une manière épigénétique: une contre-découverte importante à la thèse selon laquelle tout serait déposé dans nos gênes et donc irréversible ! Les

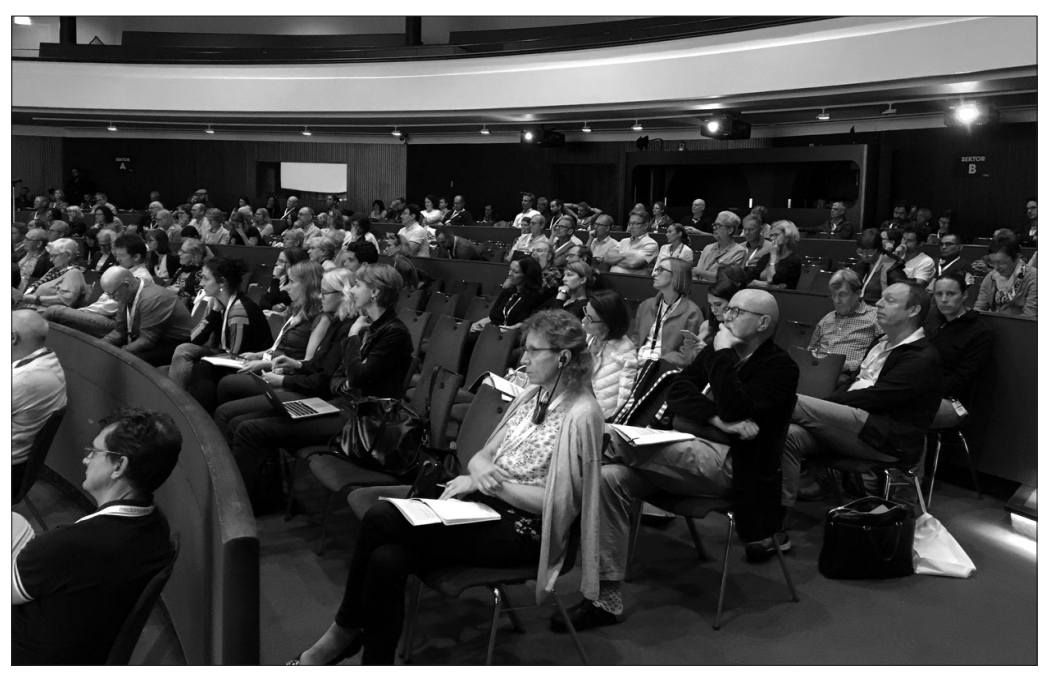

Audience intéressée

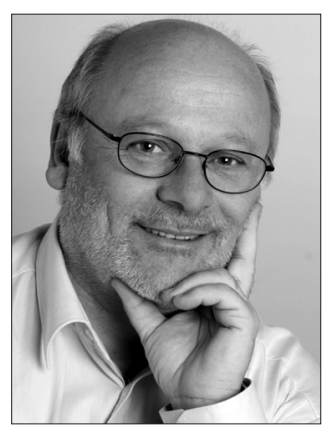

à jour! Évolution de la profession de psychothérapeute $8:$ Actualité 51 
expériences relationnelles aussi dans une psychothérapie sont de tels facteurs environnementaux pouvant contribuer à ce que les prédispositions génétiques aient ou non un effet.

Un autre exposé en plénière fut instructif. Jens Gaab, Bâle, a donné un titre spécial à son allocution sur létat de la recherche en psychothérapie : « La pointe de l'iceberg et le reste - d'oiseaux disparus, Benjamin Franklin et Stradivarius et ce que cela a à voir avec la psychothérapie». Il a parlé des facteurs d'efficience supposés depuis l'apparition de la psychothérapie avec la théorie du magnétisme de Mesmer jusqu'à aujourd'hui, il a parlé des résultats de la recherche sur les placébos et les a présentés à cet égard avec la psychothérapie. Qu'est-ce que le verum en psychothérapie? Qu'est-ce que le placébo ? Il critique la recherche psychologique dans le sens que souvent seule la pointe observable, visible de l'iceberg est analysée, la grande partie passant inaperçue. Dans ce cadre, le Titanic aurait bien coulé en raison de la collision avec la partie invisible, et non en raison de la pointe de l'iceberg, a-t-il déclaré d'une manière métaphorique. Un article spécial dans la rubrique "Focus » de ce cahier est également consacré à ses déclarations. En plus des six allocutions principales, il y a aussi eu dix conférences «State of the Art» et des manifestations parallèles avec des conférences, ateliers ou symposiums et panels sur d'innombrables sujets. Les manifestations étaient dans l'ensemble d'un bon niveau.

Pendant tout le Congrès, un thème particulier s'est retrouvé dans les manifestations quotidiennes Open-Space qui ont tenu compte du fait que ce colloque PSY nétait pas seulement le congrès annuel des psychiatres suisses, mais avait été aussi organisé par les associations AFP, FSP et la SBAP.

La question centrale était: "Psychiatrie ET psychologie: Coopération ET concurrence en faveur des soins de personnes malades psychiquement - comment l'organisons-nous concrètement ? » Malheureusement, ces manifestations Open-Space ont obtenu peu d'attention et de visites. Lors d'une manifestation plénière, des résultats ont été communiqués et un panel final a été tenu avec des représentant(e)s des associations.

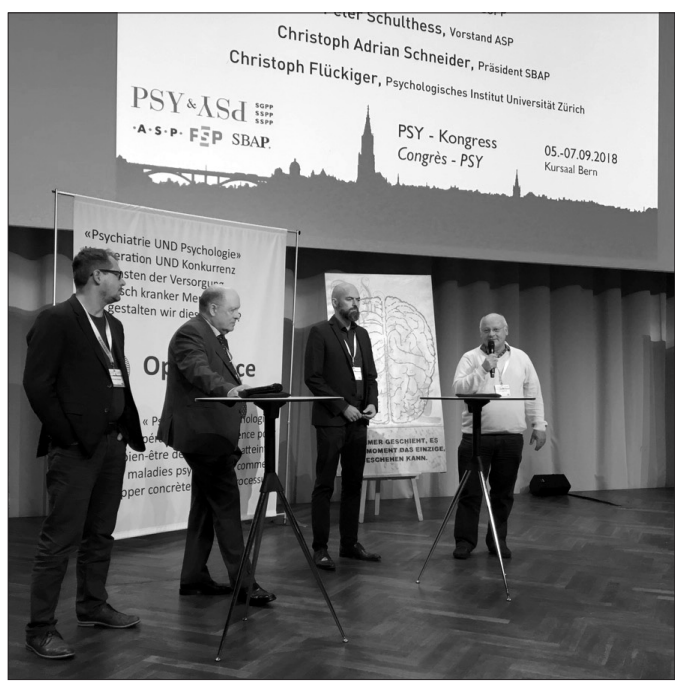

(de la gauche): Christoph Flückiger (FSP/Uni Zurich), Pierre Vallon (SSPP), Christoph Adrian Schneider (SBAP), Peter Schulthess (ASP)

Les patient(e)s ont pour leur part exigé de pouvoir entrer en contact avec des spécialistes et obtenir un accès facile à la psychothérapie, en tant que travail dans une expérience relationnelle et pas seulement être gavé de pilules. La SSPP a, quant à elle, souligné à quel point les psychiatres tiennent à la collaboration avec les psychologues et que cela fonctionnerait très bien aussi dans la pratique via la thérapie déléguée. Les associations de psychothérapie ont déploré qu'il faille un pied d'égalité pour pouvoir coopérer d'égal à égal : Même rémunération pour la même prestation lorsqu'il s'agit de la réalisation de la psychothérapie. Le modèle de délégation avec le contrat de travail qui y associé devrait être remplacé au moins par un modèle de la prescription médicale. Les associations en psychothérapie étaient d'accord sur le fait que l'on se fait davantage entendre qu'avant et que l'impasse actuelle avec la Tarmed et la répartition des fonds au sein de la FMH, la pression des économies de Santésuisse, devait être surmontée. Les manœuvres dilatoires de l'OFS exprimées aussi par le panel décrit par Gabriela Rüttimann doivent cesser avec différentes mesures: voie juridique, pétition, nouveau modèle d’assurance, manifestations.

Peter Schulthess est membre du conseil d'administration de l'ASP. 